\author{
Aus der Abtheilung für Hautkranke des städt. Krankenhauses \\ zu Dortmund.
}

\title{
Ueber einen eigenthümlichen Fall von Dermographismus.
}

(Urticaria chronica, factitia, haemorrhagica.)

Von

\author{
Dr. med. J. Fabry,
}

Oberarzt,

In seiner vorzüglichen Monographie „über Dermographismus" sagt B arthelémy mit Recht: "tous les urticariens vrais ne sont pas dermographiques, tous les dermographiques sont loins d'être urticariens." Unter diesem Gesichtspunkte sei die Krankengeschichte eines, wie wir glauben, ungewöhnlichen Falles von Urticaria factitia mitgetheilt; die Diagnose bezüglich der Hautsymptome konnte nach unserem Dafürhalten nicht anders lauten, wenngleich, wie von vornherein bemerkt werden soll, die Hauterkrankung wahrscheinlich das Symptom einer schweren Allgemeinerkrankung war; somit dürfte der Fall auch gleichzeitig einen Beitrag darstellen zu dem Capitel der Beziehungen der Hautkrankheiten zu Allgemeinerkrankungen. Was die Bezeichnung Dermographismus anlangt, muss Kaposi vollkommen beigepflichtet werden, der sagt: "Es war ganz unpöthig, mit der femme autographique von Dujurdin-Beaumetz ein solches Aufsehen zu machen, als wäre dies etwas ganz Unerhörtes und den Ausdruck "Dermographismus" in die Dermatopathologie einzuführen, als bezeichnete dies eine besondere Krankheit." Damit bleibt ja bestehen die Trennung zwischen Urticaria spontanea und factitia; zu letzterer möchten wir also den von uns beobachteten Fall rechnen. 
Den Fall beobachteten wegen der Seltenheit der Hautsymptome und wegen der Eigenthümlichkeit des gesammten Krankheitsbildes gleichzeitig mit mir eine Reihe Bochumer Collegen; die später aufgenommenen anamnestischen Daten verdanke ich einem derselben, dem Collegen Dr. L ind in Bochum.

\section{Krankenbericht.}

Anna Reinhard, geb. den 16./II. 1835 in der Nähe von Eisenach. Die Eltern und Geschwister der Patientin haben keine Hautausschläge gehabt, waren alle gesund; sie selbst war immer gesund, that schwere Arbeit auf dem Lande und beim Vieh, heiratete im Alter von 25 Jahren anno 1860. Da die Patientin im Jahre 1898 starb, so war sie also 38 Jahre verheiratet. Auch in der Ehe war sie von guter Gesundheit, hat nur einmal eine leichte Lungenentzündung durchgemacht. Patientin hat 7 ausgetragene Kinder geboren, von denen noch 4 leben, hat niemals abortirt; zwei Kinder starben im frühesten Kindesalter; woran sie starben, ist nicht zu erfahren; ein Kind starb mit 17 Jahren an Pleuritis nach 17tägiger Krankheit. Für Tuberculose ergibt die Anamnese nicht die geringsten Anhaltspunkte, auch Lues ist vollständig auszuschliessen. Der Mann der Pat. ist Bergmann und lebt trotz der grösseren Kinderzahl in durchaus geordneten Verhältnissen; Nahrungssorgen waren in der Familie nicht vorhanden, auch war die Lebensweise der Familie nicht von der gewöbnlichen abweichend und bestand die Nahrung vorwiegend in gekochtem, weniger in gesalzenem Fleisch. Scorbut war überhaupt in der Gegend nicht beobachtet.

Die Hauterkrankung begann bei Pat. Mitte des Jahres 1895, also stand die Pat. im Alter von 60\% Jahren. Das erste, was beobachtet wurde, waren Sugillationen in den oberen Augenlidern, die mit Dauer von 14 Tagen bald kamen, bald schwanden, ohne besondere Beschwerden zu machen; dann stellte sich Jucken am Halse und auf der Brust ein, was Pat. zu kratzen veranlasste; die Folge des Kratzens war, dass blutige, lange Zeit wochenlang bestehen bleibende Striemen entstanden; diese Striemen verschwanden ganz allmälig unter Bildung der bekannten Farbenscala, wie sie bei $H$ a utblutungen beobachtet wird.

Schleimhautblutungen aus den Respirationsorganen oder dem Verdauungstractus, endlich aus den Harnwegen sind bei Pat. während der ganzen Dauer der Erkrankung nicht beobachtet worden.

Die Hauterscheinungen nahmen mit der Zeit immer zu, alte Flecken verschwanden, neue traten hinzu.

Fast genau 1 Jahr vor dem Tode der Patientin trat ein ganz kolossales Oedem der Zunge ein, das sich nicht wieder zurückbildete, im Gegentheil allmälig immer voluminöser wurde; durch den damit verbunden vermehrten Speichelfiuss und die sehr erschwerte Nahrungs- 
aufnahme machte dasselbe der Patientin sehr viele Beschwerden; wir kommen darauf bei Wiedergabe des objectiven Befundes eingehender zurück. Die Pat. starb, 63 Jahre alt, an Schwäche; das Sensorium war bis kurz vor dem Exitus vollstāndig frei.

Der zuerst behandelnde Arzt musste sich, als er die Pat. zum ersten Male untersuchte, sagen, dass er ein gleiches oder auch nur ähnliches Krankheitsbild noch nicht gesehen hatte; damals waren also die Erscheinungen auf der Haut das auffallendste Symptom, während an der Zunge das Oedem sich noch nicht entwickelt hatte; es kann daher gar nicht Wunder nehmen, dass zu einer Zeit, wo die Lepra so ausserordentlich viel, durch die Memeler Fälle veranlasst, von sich reden machte, von zuerst den Fall beobachtenden Aerzten an letztere Erkrankung gedacht wurde. Warum sollte sich bei der Arbeiterbevölkerung des hiesigen Industriebezirkes, die sich zum grossen Theil durch Zuwachs aus den östlichen Provinzen recrutirt, nicht auch Fälle von Lepra einschleppen? Allerdings fehlt es bis heute noch an der ersten Beobachtung, aber mit der Möglichkeit hat man immer zu rechnen.

Alle therapeutischen Massnahmen blieben ohne den geringsten Einfluss auf den Verlauf der Erkrankung.

Verfasser hatte etwa 1/2 Jahr vor Eintritt des Exitus letalis Gelegenheit Patientin zu untersuchen und muss gleichfalls gestehen, dass er ein derartiges Krankheitsbild noch nicht beobachtet hatte. Die Pat. lag zu Bett und war bereits recht elend und abgemagert. Die auffallendste Erseheinung, die dem Aussehen der Pat. geradezu etwas Schreckhaftes gab, war das kolossale Oedem der Zunge; das Volumen der Zunge war mindestens um das $\mathrm{F} u ̈ \mathrm{nffache}$ vergrössert und hing dieselbe weit am Munde heraus; es erinnerte der Mund, man möge den Ausdruck verzeihen, an die spitz zulaufende "Schweineschnauze; die arme Pat. war nicht in der Lage, die vergrösserte Zunge auch nur für kurze Zeit in die Mundhöhle zurückzubringen und litt natürlich durch den vermehrten Speichelfluss und überhaupt durch den unerträglichen Zustand entsetzlich. Die Vergrösserung der Zunge war derart, dass die längliche Form der Zunge beibehalten war, die Zunge war in toto vergrössert, keine Lappenbildung abgesehen von den 
Stellen, wo die Zähne mechanisch Einkniffe gemacht hatten; die Oberfläche war sonst ganz normal, nirgends waren Erosionen vorhanden, ebenso im Gewebe keine Blutungen in Gestalt von Petechien oder grösseren Blutherden. Die Consistenz war teigig, Druck absolut nicht schmerzhaft. Submaxillare Drüsen nicht geschwollen. Auch von anderer Seite wurde Pat. wie von uns vorgeschlagen, den qualvollen Zustand durch Vornahme einer Keilexcision erträglicher gestalten zu lassen, jedoch war Pat. nicht dazu zu bewegen.

Als Pat. bei weiterer Untersuchung nun die Brust und den Rücken frei machte, fielen auf der Schulter beiderseits unregelmässige, tief dunkelpurpurrotheStriemen auf; ebenso an der Brust, in der Bauch- und Rückengegend ganz unregelmässig gestaltete Flecken, die frischeren von tief dunkelrother Farbe, andere in den bekannten Differenzen der Blutextrarasate bis zum Grünlichen und Gelblichen. Pat. trug iber den Schultern zum Halten des Unterrocks eine Art Träger, und es war unverkennbar, dass der leichte Druck derselben das Exanthem hervorgerufen hatte; dieser Umstand liess uns natürlich vermuthen, dass es sich um ein artificielles traumatisches Exanthem handle; diese Vermuthung wurde sofort bestätigt, indem es gelang durch Ueberstreichen mit einem stumpfen Gegenstand, beliebige Figuren a uf dem Rücken sowie am ganzen Körper hervorzurufen; also die bekannte Urticaria factitia oder der Dermographismus lag vor. Aber es war nicht die gewöhnlich beobachtete Form. Der Umstand, dass mit der gew öhnlichen Leisten- oder Quaddelbildung sogleich Blutungen eintraten, gab dem Hautphänomen ein Gepräge, das den Fall entschieden bemerkenswerth macht. Während die quaddelartigen Erhebungen über das Niveau verhältnissmässig bald zurückgingen, schon nach Minuten, blieben die purpurrothen Streifen bestehen, nach Angabe der Patientin, der Angehörigen und der mit beobachtenden Collegen wochenlang, um ganz allmälig im Kreislauf der bekanntenRegenbogenfarbeninnerhalb 2-4 Wochen ungefähr zu verschwinden, ohne Pigmentirungen zu hinterlassen, wie etwa bei der Urti- 
caria pigmentosa. ${ }^{1}$ ) Es sind also bei der Patientin s p on ta $\mathrm{n} k$ e in e Flecken aufgetreten, sondern sie wurden ausgelöst durch Traumen; allerdings genügte schon der einfache leichte Druck der Kleidung oder aber Kratzen.

Die Untersuchung der Brust- und Bauchorgane liess nichts Abnormes nachweisen; ausser hochgradiger Anämie fand sich nichts; die Untersuchung des Blutes auf zellige Bestandtheile scheiterte gleichfalls an dem Widerstande der Pat., die wohl zur Einsicht gekommen war, dass ärztliche Kunst ihr nicht zu helfen in der Lage war.

Geistig machte Pat. einen vollständig normalen Findruck, ebenso fand sich keine Abnormität des Nervensystems; mässige multiple, indolente Schwellung der Leisten-, Axillar- und Nackendrüsen.

Leider war es auch nicht möglich Patientin zu bestimmen, sich ein Stückchen Haut zur histologischen Untersuchung excidiren zu lassen; das hätte theoretisch gewiss grosses Interesse gehabt, insofern, als sich vielleicht Veränderungen an den Gefässen hätten nachweisen lassen; praktisch kann nichtsdestoweniger nicht im Geringsten daran gezweifelt werden, dass durch das Streichen mit einem stumpfen Gegenstand, nicht nur auf der Haut die Quaddelbildung, sondern auch die Blutaustritte in die Haut hervorgerufen wurden. Somit konnte die Diagnose in der That nur lauten: Urticaria chronica, factitia, haemorrhagica.

Dass es in der That eine Urticaria war, dafür spricht der Umstand, dass die Primärefflorescenzen echte Quaddelbildungen sind mit der bekannten weissen Porzellanfarbe auf der Höhe des Niveaus. Da der Krankheitsprocess sich über fast 3 Jahre hinerstreckte und von Anfang an bis zum Eintritt des Todes die Hauterscheinungen dieselben blieben, so kann man

1) Bekanntlich sind von Pi ck und mir auch bei der Urticaria pigmentosa xanthelasmoides Haemorrhagien nachgewiesen worden, während sich in allen anderen Fällen der Mastzellentumor Unna's fand; eine jüngst aus der Kaposi'schen Klinik erschienene Arbeit über Urticaria pigmentosa macht daher mit Recht den Vorschlag, die beiden Fälle mit Haemorrhagien abgesondert zu lassen. (Kreibich, Ueber Urticaria chronica, Arch. f. Dermat. Bd. XLVIII, 163.) 
füglich von einer chronischen Form der Erkrankung sprechen. Spontan sind während der ganzen Dauer des Krankheitsverlaufes Efflorescenzen nicht aufgetreten, sondern bei allen lässt sich als Ursache ein Trauma nachweisen, allerdings genügte schon der einfache Druck der Kleidung; auf der anderen Seite war es jederzest möglich, an jeder beliebigen Körperstelle beliebige Figuren zu beschreiben. Diese Eigenthümlichkeit hatte auch die Haut an den Fusssohlen und den Volarflächen der Hände. Geschickt oder sagen wir raffinirt ausgenützt, hätte der Fall wohl in andern Gegenden als Stigmatisation Aufsehen erregen können, hierzulande fehlt für den Wunderglauben der Nährboden. Uebrigens fand ein Transsudiren von Blut oder blutig gefärbter Flüssigkeit an den gereizten Stellen nicht statt. Dass der Zusatz "haemorrhagica" trotz Fehlens mikroskopischer Untersuchungen ein wohlbegründeter ist, kann auch wohl kaum angezweifelt werden; wir verweisen nochmals auf die im Krankenbericht erzählten Thatsachen, vor allem darauf, dass die Quaddelerhebungen sich nach einigen Minuten zurückbildeten, während im Bereich derselben die purpurrothenstreifen persistent blieben und sich im Verlauf von Wochen unter den Farbenerscheinungen, wie sie bei traumatischen Hämorrhagien, beispielsweise bei der bekannten "Blutbeule" beobachtet werden, zurückbildeten. Für uns ist es auch wahrscheinlicher, dass so starke Blutungen nicht per diapedesin sondern per rhexin in Folge Brüchigkeit der kleinsten Gefässe der Cutis und Subcutis entstanden sind. Nach Unna's Ermittelungen zeigt sich bei der gewöhnlichen Urticaria factitia ein in allen seinen Theilen hyperplastischer Gefässbaum; wir müssen demnach für unseren Krankheitsfall einen gesteigerten Process an den kleinsten Gefässen annehmen, der za einer abnormen Brüchigkeit derselben gefuihrt hat.

War die Urticaria eine genuine oder das Symptom einer Allgemeinerkrankung? Die Anamnese und der Krankheitsverlauf hat zwar ausser der hochgradigen Anämie keine Anhaltspunkte gegeben; zwar hat Pat. eine leichte Lungenentzündung gehabt und ein Kind ist anPleuritis gestorben, aber im Uebrigen lässt die Anamnese auf Tuberculose vollständig im Stich; nichtsdestoweniger möchten wir also zu der Annabme hinneigen, 
dass eine schwere Allgemeinerkrankung vorgelegen hat schon in der einfachen Erwägung, dass die Urticaria eigentlich niemals eine üble Prognose hat. Die Primärsymptome waren so charakteristisch, dass darnach dem Falle eine andere Deutung wie Urticaria nicht gegeben werden konnte; differentiell diagnostisch musste in erster Linie an Purpura haemorrhagica gedacht werden. Die Schwellungen der oberen Lider zu Beginn der Erkrankung waren wahrscheinlich gleichfalls hämorrhagischer Natur; das später auftretende hochgradige stabile Oedem der Zunge ist gewiss eine sehr merkwürdige Erscheinung, kann aber an der Diagnose Urticaria nichts ändern. Es braucht wohl kaum hervorgehoben zu werden, dass gerade die ungeheure Volumszunahme der Zunge durch Behinderung der Nahrungsaufnahme mit ein wesentliches Moment war für das Eintreten des Exitus letalis in Folge von Entkräftung. Section ist nicht bewilligt worden. 


\section{Literatur.}

1. Dujardin Beaumetz, La femme autographique. Annales de Derm. 1880.

2. Caspary, Archiv für Derm. 1882. pag. 719 .

3. Michelson, Ueber Urticaria factitia etc. Berliner Klinische Wochenschrift 1884. Nr. 7. u. 8.

4. Unna, Beiträge zur Anatomie der Urticaria simplex und pigmentosa, Monatshefte, Ergänzungsheft 3, 1877.

5. Barthelémy, Du Dermographisme. II. internationaler derm. Congress Wien 1892. In dieser Abhandlung findet sich die ausführliche Literatur. Benutzte Lehrbücher: 1. Sim on, Lehrb. der Hautkrankheit. Berlin, 1851. 2. Hebra und Ka posi, Lehrb. der Hautkrankh., Erlangen, 1874. 3. Ne uman n, Lehrb. der Hautkrankheit., Wien, 1876. 4. Jo sef, Lehrb. der Hautkrankheit., Leipzig, 1892. 5. Unna, Histopathologie, Berlin, 1894. 6. Kromayer, Allgemeine Dermatologie, Berlin, 1896. 7. Kaposi, Lehrb. der Hautkrankheit., Wien, 1899. 8. Ja ri s ch, Lehrb. der Hautkrankheit., Wien, 1900. 